Research Article

\title{
Elder Orphans Hiding in Plain Sight: A Growing Vulnerable Population
}

\author{
Maria T. Carney, ${ }^{1}$ Janice Fujiwara, ${ }^{1}$ Brian E. Emmert Jr., \\ Tara A. Liberman, ${ }^{1}$ and Barbara Paris ${ }^{2}$ \\ ${ }^{1}$ Long Island Jewish Medical Center, Northwell Health, Hofstra Northwell School of Medicine, New Hyde Park, NY 11040, USA \\ ${ }^{2}$ Maimonides Medical Center, Icahn School of Medicine at Mount Sinai, Brooklyn, NY 11219, USA \\ Correspondence should be addressed to Maria T. Carney; mcarney@northwell.edu
}

Received 1 February 2016; Revised 16 June 2016; Accepted 12 July 2016

Academic Editor: Iracema Leroi

Copyright (c) 2016 Maria T. Carney et al. This is an open access article distributed under the Creative Commons Attribution License, which permits unrestricted use, distribution, and reproduction in any medium, provided the original work is properly cited.

\begin{abstract}
Adults are increasingly aging alone with multiple chronic diseases and are geographically distant from family or friends. It is challenging for clinicians to identify these individuals, often struggling with managing the growing difficulties and the complexities involved in delivering care to this population. Clinicians often may not recognize or know how to address the needs that these patients have in managing their own health. While many such patients function well at baseline, the slightest insult can initiate a cascade of avoidable negative events. We have resurrected the term elder orphan to describe individuals living alone with little to no support system. Using public data sets, including the US Census and University of Michigan's Health and Retirement Study, we estimated the prevalence of adults 65 years and older to be around 22\%. Thus, in this paper, we strive to describe and quantify this growing vulnerable population and offer practical approaches to identify and develop care plans that are consistent with each person's goals of care. The complex medical and psychosocial issues for elder orphans significantly impact the individual person, communities, and health-care expenditures. We hope to encourage professionals across disciplines to work cooperatively to screen elders and implement policies to prevent elder orphans from hiding in plain sight.
\end{abstract}

\section{Introduction}

It is common for physicians who provide care to older adults to encounter an elder orphan in their office, hospital, or an emergency room, but they do not recognize them as such or identify the risks related to this. We define elder orphans as aged, community-dwelling individuals who are socially and/or physically isolated, without an available known family member or designated surrogate or caregiver. This demographic, those aging alone with limited support, is expected to increase as the United States population continues to age and people live in the community with more chronic illnesses. Recent national media reports have also brought attention to this growing problem [1-3]. Thus, we strive to raise awareness of the concept of aging alone without an available caregiver and introduce the term elder orphan to more clearly define this vulnerable population and identify these individuals as high risk in an effort to call to action health-care providers, government agencies, and general public to address their needs and minimize preventable illness. We also provide guidance on how to screen and care for an individual who may be at risk for being an elder orphan.

Below, two case scenarios are described which underscore the concepts and risks involved with elder orphans. These cases highlight the crucial need to identify members of this population in order to prevent medical crises.

Case 1 (Ms. H. M.). Ms. H. M. is a 92-year-old widow living in her home with her 65-year-old son with cerebral palsy, who is dependent upon her. She has managed to live at home with little help for many years. Over the past few months, however, she noticed that her function is declining; she is becoming unable to drive or even do many household chores. Moreover, because of a growing lethargy, she is finding it more difficult to even cook and clean. A fiercely independent woman, she 
has attempted to hire aides, but she promptly lets them go because of difficulty supervising them.

After meeting with a social worker while still able to make decisions and through an introduction to legal guidance in the community, a plan of action was determined for both her and her son, as well as the beginnings of preparations of what would happen with her son if anything were to happen to her. A distant but willing family member was reconnected and helped support the plan created. Through identification of her and her son's risk to be "orphaned" and the creation of a care-giving plan and identification of a health-care proxy or surrogate decision-maker, the likelihood of medical catastrophe for this elder orphan and her son (who will eventually inherit elder orphan status) has decreased significantly.

Case 2 (Mr. H. B.). Mr. H. B. is a 72-year-old man living alone in his apartment in Long Island, New York. He was admitted to a Palliative Care Unit for complex medical, social, and wound care after a failed suicide attempt, having slit his wrists with a razor. Upon admission, it was found that Mr. H. B. was never married and was childless and his closest relative was residing in California, thus uninvolved in his care. Mr. H. B.'s relative had little knowledge of his condition. Once wound care was complete, finding placement for Mr. H. B. was difficult, as he was not healthy enough to travel to California to be near his only relative, nor was he psychologically or medically well enough to be discharged home alone. With no known caregiver identified, after a several-week stay in the hospital, he was eventually relocated to a skilled nursing facility for further wound care with a long-term plan to be relocated near his only relative in California.

The term elder orphan was utilized on rounds with Case 2 to highlight the vulnerability of individuals with limited to no support in the community whose abilities are being challenged and risk of losing independence is significant. This particular case led to much discussion and academic interest because of an additional perceived increase in individuals being seen at the hospital who lack care-giving and decisionmaking support by spouse, partner, family, or community.

For patients like Ms. H. M. and Mr. H. B., we utilize the term elder orphan. It is imperative that the medical and social community become more familiar with this term as it highlights a population aging alone without a caregiver and with significant barriers to care. Furthermore, the term elder orphan when utilized properly creates an important notification to health-care providers that care-giving needs are lacking and are an important aspect to treatment. Moreover, we expect the prevalence of those aging alone and those who are at risk of being elder orphans to continue to increase as individuals are living longer, with multiple chronic diseases, alone, and geographically distanced from other family members. Thus, in this paper, our goals are threefold: to evaluate the terms synonymous with aging alone or "elder orphan" use in literature, identify the prevalence of being at risk to be elder orphans and the risks facing this population, and provide guidance when faced with caring for an elder orphan.

\section{Methods}

2.1. Literature Search. A literature search was undertaken to examine the use of the term elder orphan or any term synonymous with age, isolated, and/or alone. To better characterize this vulnerable population and identify clinical correlates for risk factors, four databases were searched: PubMed, Google Scholar, Health Reference, and CINAHL. Reviews of police and emergency management department programs, U.S. Census data, and the North Shore-LIJ Health System social work database were also conducted to assist in terminology use for vulnerable adults. The search terms utilized included elder orphan, unbefriended elder, patients without surrogates, vulnerable elderly, social isolation, loneliness, childless unmarried, frail elderly, lone elders, and aging alone as shown in the following list (synonyms encountered in reviewing the medical literature on social isolation in older adults).

Similar terms encountered while searching "elder orphan" are the following:
Aging alone.
Elder orphan.
Frail elderly.
Patients without surrogates.
Social isolation.
Unbefriended elder.
Vulnerable elderly.

A total of 56 publications were identified and reviewed from international medical, legal, and lay press sources dating back approximately 35 years (Table 1 ).

2.2. Prevalence. Estimates of the prevalence of elder orphans living in the United States were determined by using previously published, valid, and publicly accessible national surveys. Initially, we conducted an analysis of U.S. Census data. We then turned our attention to the Health and Retirement Study (HRS) [4]. The HRS is sponsored by the National Institute on Aging (Grant number NIA U01AG009740) and is conducted by the University of Michigan. It surveyed a representative sample of over 22,000 people in the United States aged 65 years and older about aspects of their personal life and family. We recoded and parsed the data so as to examine marital status, number of children, number of children in contact, number of children in close proximity, number of siblings, and number of siblings in close proximity of the subject. From this analysis, we devised a spectrum of categories which, by definition, can lead to aged, isolated, alone status. We then extrapolated the prevalence (in percent of the population) for each tier in the spectrum, using the numbers derived from the HRS, and then further estimated the prevalence of at-risk elder orphans in the general population.

\section{Results}

\subsection{Literature Search}

3.1.1. Use of Term. Through the literature search efforts the term elder orphan was found to be first designated by Kunerth 


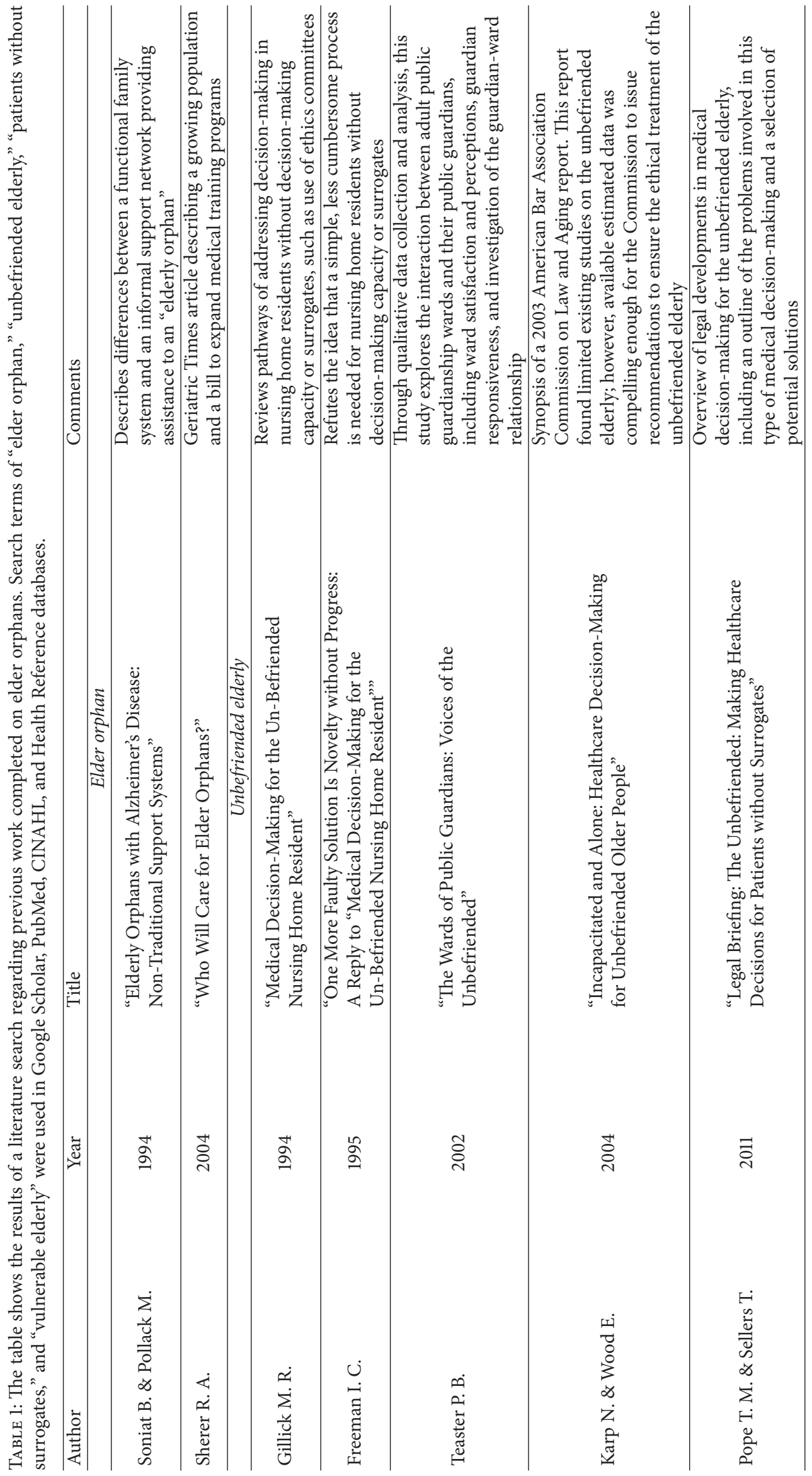




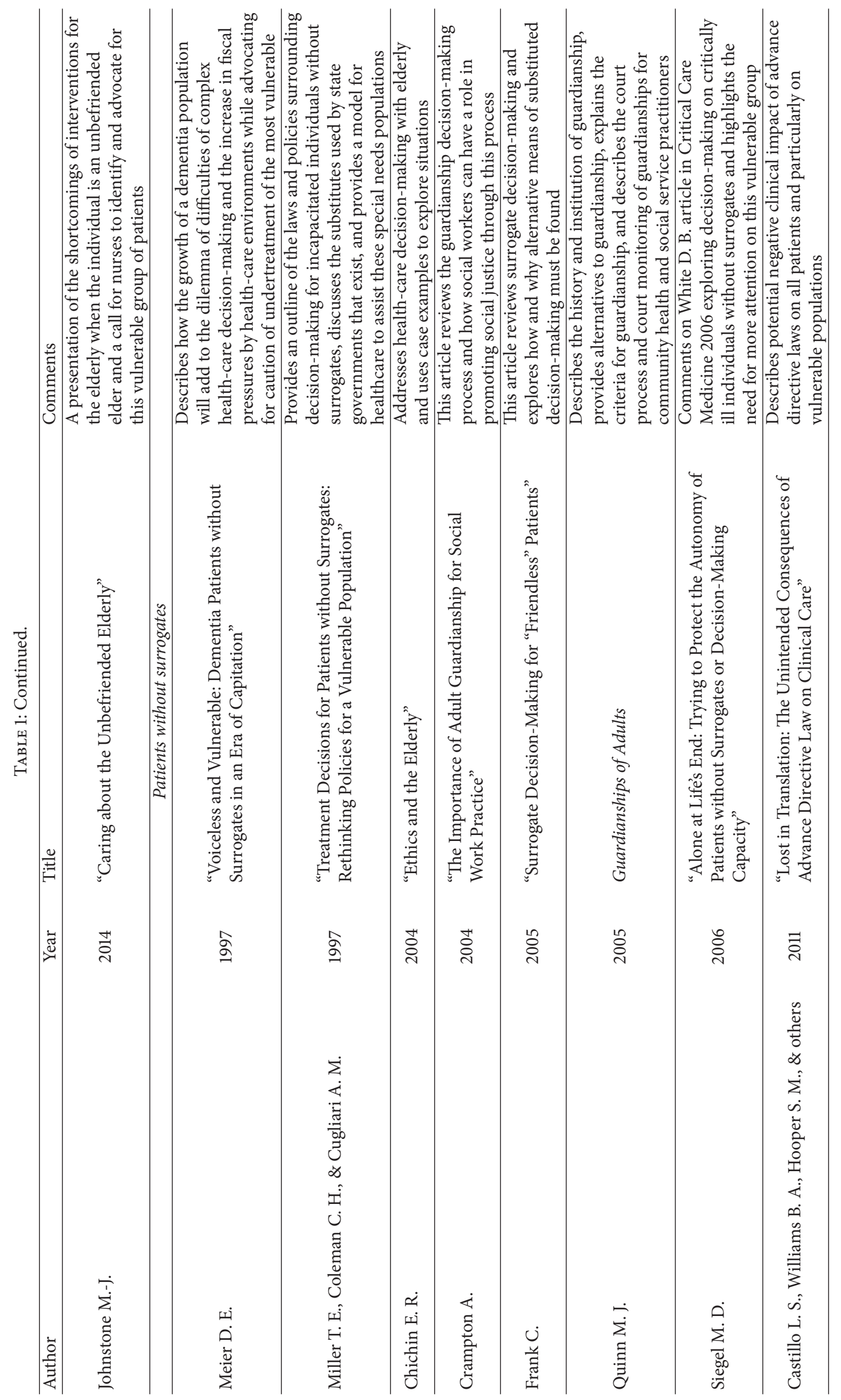




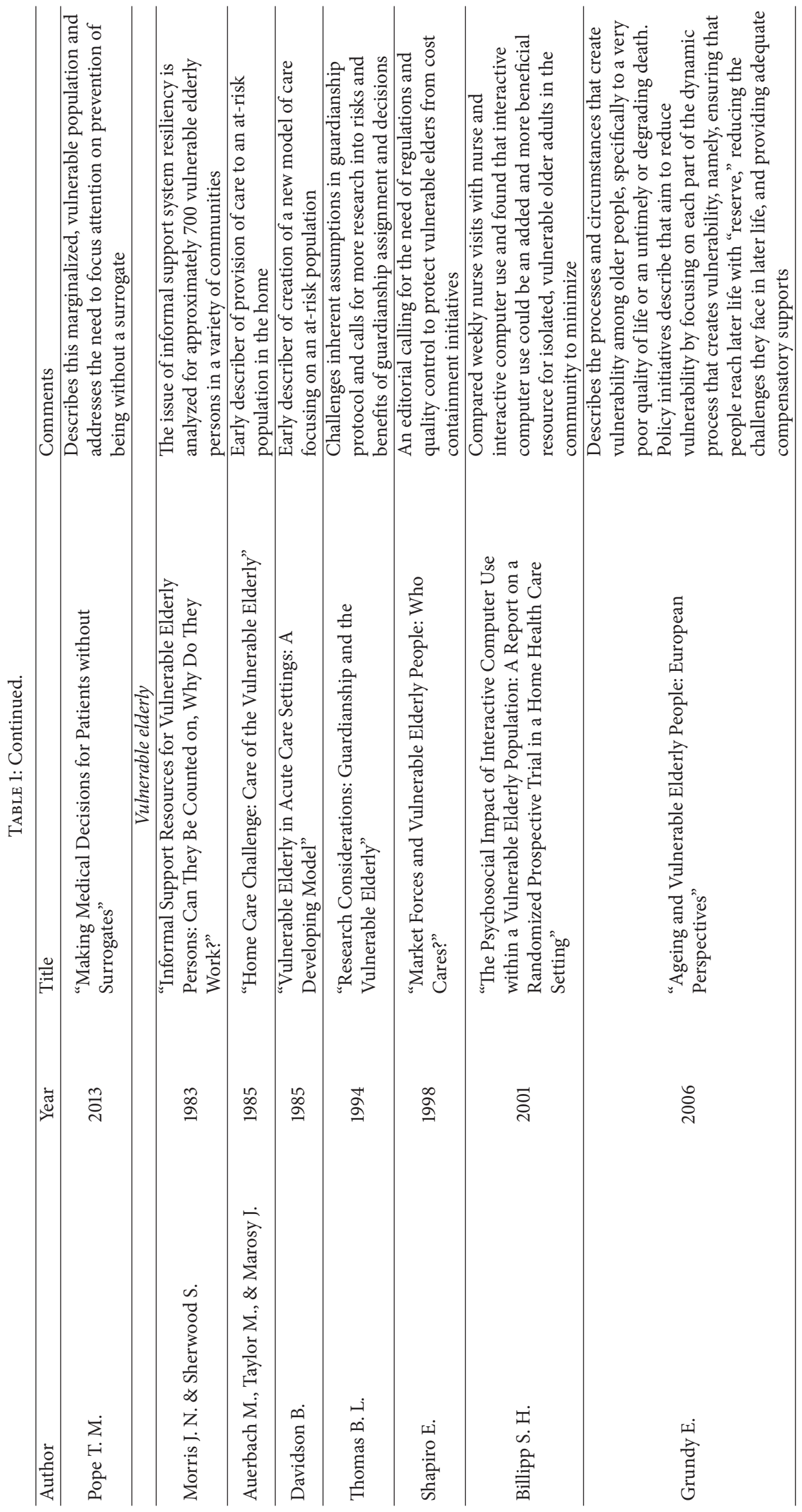




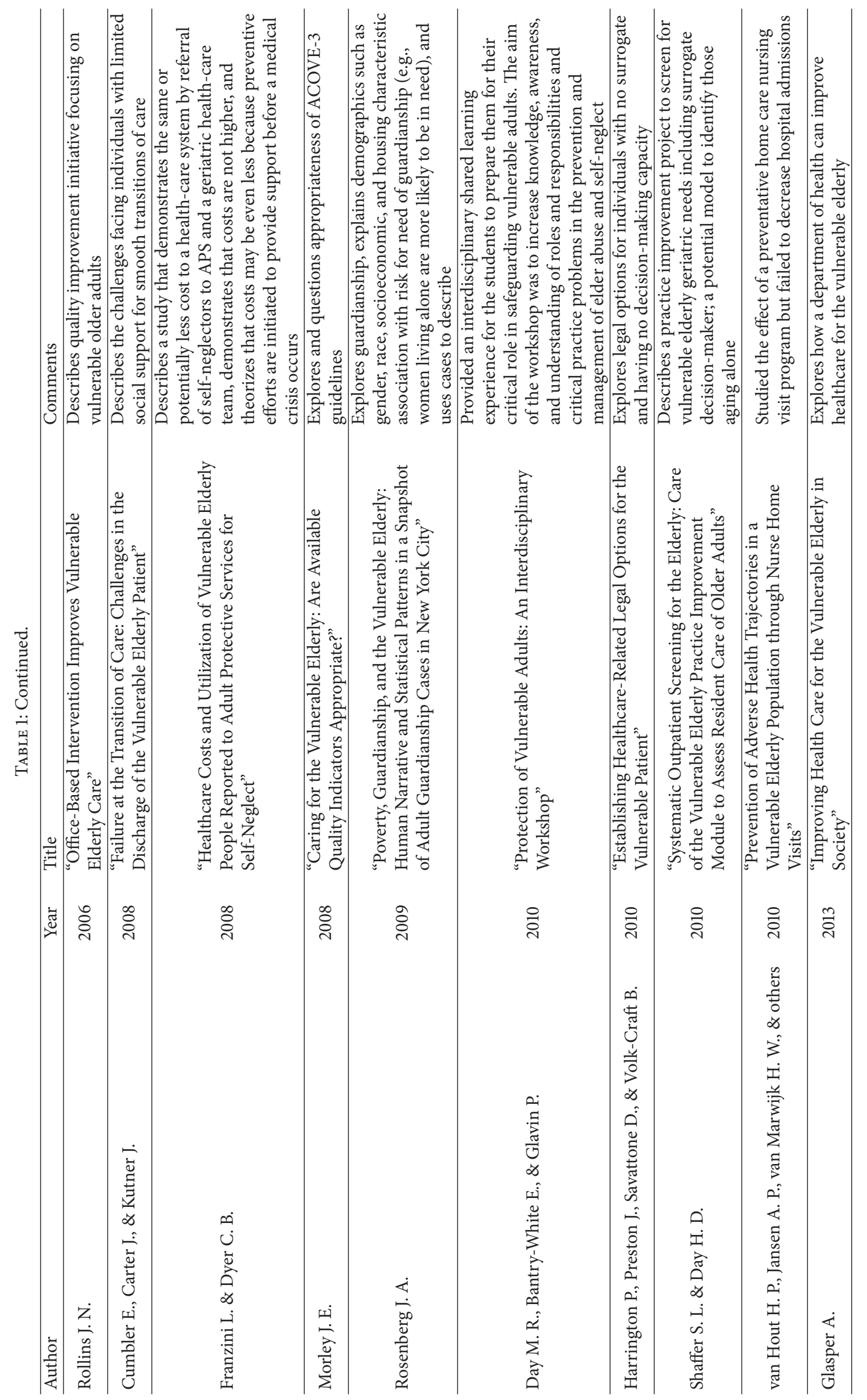


[5] in 2003 and Sherer [6] in 2004 in the lay press, and then it resurfaced in a state nursing journal in 2005 in a didactic article by Varner [7] to describe a growing subset of the geriatric population that requires special consideration. Since then, the term has been dormant, and as a consequence pertinent concepts related to the elder orphan, such as the estimated prevalence of and risks associated with being an elder orphan, are not well documented in the medical literature.

We propose that this term be resurrected out of dormancy in order to highlight a need to intervene for social support with a goal of minimizing adverse medical events and frailty. Elder orphans are a unique subset of the aging population, as their inclusion in this category is often due to circumstance rather than choice. As independent individuals, they have functioned sufficiently well on their own and thus do not actively plan for their medical future. As they age and decline, they realize, often too late, that they can no longer complete many of the tasks that they were previously able to do. Stemming from this inability, elder orphans may no longer access the care that they need, and acute, possibly preventable, medical events occur that can easily lead to hospitalization. These events often incur significant costs to the health system and undue suffering to the patient. By raising awareness for this group of aging adults by referencing them with a benevolent and informative title such as elder orphan, we hope this group will get more attention by the medical and social community. Advanced planning and consciousness will be raised for these individuals, and with coordination between medical and community organizations they can be directed to appropriate services before their function declines, facilitating maintenance of quality of life in their own communities for as long as possible.

The term elder orphan raises clear awareness to medical providers of the vulnerability of the individual and the importance of managing the patient's care comprehensively and multidisciplinarily.

\subsection{Risks}

3.2.1. Social Support. In addition to the likelihood of not receiving adequate care, being at risk for elder orphan status (aged, community-dwelling individuals who are socially and/or physically isolated, without an available known family member or designated surrogate or caregiver) can have a series of adverse biopsychosocial consequences on an individual. Low social support has been linked to both poor physical and psychological health and an increased risk of mortality for the elderly population $[8,9]$. Moreover, decreased social interaction that can stem from this lack of support is correlated with low affect and arousal [10], poor cognitive and social skills [11], and altered neurophysiological functioning [12].

3.2.2. Isolation and Loneliness. Isolation and loneliness are distinct in that isolation is the objective state of having minimal contact with others, whereas loneliness is the subjective feeling of being socially alone and isolated. Both of these states have been identified as risk factors for physical and cognitive decline.
Perissinotto et al. [13] completed a longitudinal cohort study of 1604 subjects and found that among those who are 60 years of age and older loneliness was a predictor of both functional decline (in areas including mobility, climbing, upper extremity tasks, and activities of daily living) and death. Additionally, Sorkin et al. [14] found that greater levels of loneliness and lower levels of emotional support and companionship were correlated with an increased risk of coronary disease.

Social isolation has been shown to be a risk factor for medical complications and mortality. Wenger et al. [15] studied working class individuals and found that social isolation is correlated with advancing age; being male and single; living alone; and having no children. These researchers also found an association between social isolation and retirement migration (moving to a new area upon retirement), poor health, restricted mobility, admission to institutional care, low morale, poor rehabilitation, and mental illness.

In a study of 271 community-dwelling elderly women, Thompson and Heller [16] found that both subjectively and objectively isolated women had poorer psychological wellbeing than the population mean. Moreover, those who were objectively isolated comorbidly exhibited poorer functional health. Finally, Udell et al. [17] found that in an international outpatient population with atherothrombosis living alone was associated with both increased cardiovascular death and four-year mortality, a trend which was found to grow stronger as the population aged.

3.2.3. Marriage and Children. Being married provides advantages for medical care and support for patients. In an analysis of childless elderly patients discharged from a hospital, marital status was found to be a major determinant of the level of support the patient received after discharge. Although childless, married individuals tended to rely solely on each other and thus were more socially isolated, they were resourceful in using long-term accumulation of social resources to meet their needs [18].

Childlessness is an important risk factor for social isolation. Many studies have shown that childless adults often do have support networks, usually consisting of relatives, friends, and neighbors. However, these networks are less likely to provide the long-term commitment and comparable high level of support that children offer $[19,20]$. Interestingly, evidence is inconclusive regarding the long-term difference between childless older adults and elders with children. Although the childless elderly appear to score lower on measures of objective social support, another evidence suggests that their psychological wellbeing does not significantly differ from older adults with children [21]. It is important to note that gender was a mediating factor; Zhang and Hayward found that childless men had higher rates of loneliness and depression than childless women.

An interesting concept regarding childlessness in the elderly arises when considering parents who outlive their children. The loss of a child can cause severe psychosocial stress on an individual, especially when the child dies as a result of disease. Parents may attribute the death as resulting from their actions or perceived inactions and, as part of their 
bereavement process, socially isolate themselves [22]. If the parent is older or single, this isolation can have devastating consequences on his or her health and welfare.

Another trend that may further impact adults outliving their children is described in the American Medical Association 2012 study that found that the current generation may be the first to encounter parents outliving their children. This is attributed to childhood obesity which in turn increases rates on hypertension, diabetes, stroke, and osteoarthritis upon reaching middle age. The University of Michigan's Joyce Lee found that people born between 1966 and 1985 became obese at much faster rates than previous generations [23].

3.3. Prevalence. According to 2010 U.S. Census data, nearly 19 percent of women aged 40 to 44 years have no children, as compared to about 10 percent in 1980 [24]. Furthermore, in 2009, almost one third of Americans aged 45-63 years are single, a 50 percent increase from $22 \%$ in 1980 [25]. There are no signs of this trend reversing. While being a parent or spouse does not guarantee care in old age, the bulk of America's elderly are cared for primarily by their spouses and children [26].

Limited data exists to measure the prevalence of this population. HRS data was used to estimate aging alone with limited support using marital status, having children, having siblings, or having children or siblings not in contact or not within 10 miles (existing HRS criteria and surrogate conditions deemed by authors as possible local care-giving involvement ability) (Table 2). Based on data from the HRS, we estimate that the prevalence of being at high risk for elder orphan status is to be as high as $22.6 \%$. Fortunately, we found that individuals who are most likely already elder orphans, by definition, are just a small percentage of the population (Table 2).

\section{Discussion}

We define elder orphans as aged, community-dwelling individuals who are socially and/or physically isolated and have no known family member or designated surrogate available to them. Both the safety and the independence of this demographic are threatened. With the high prevalence of individuals aging alone and the clear risks associated, it is crucial that the medical and social community become aware of this pressing issue. Moreover, the medical community must actively screen and take steps to care for individuals who fall into this demographic; consider the following lists: Questions to Screen for Risk for Elder Orphan Status and Ten-Step Guide to Caring for an Elder Orphan.

\section{Questions to Screen for Risk for Elder Orphan Status}

(i) Do you have a spouse or significant other?

(ii) Do you have children? Are they nearby?

(iii) Do you have family members or friends that help you cope with life challenges?

(iv) Do you have someone to help you make medical decisions?

(v) Do you have someone to help with bills, financial decisions? (vi) Do you have a health-care proxy or any advance directives?

(vii) Who is the person you would call upon in an emergency or crisis situation?

(viii) Do you have a home health aide to help with personal care such as bathing, dressing, and other activities of daily living?

\section{Ten-Step Guide to Caring for an Elder Orphan}

(1) Identify All Medical Issues. This may involve speaking with the patient's known providers and other personal contacts, telephoning pharmacies, and accessing old charts, laboratory work, and imaging studies. Consider asking the following:

(i) Have you fallen in the past 6 months?

(ii) Do you have 3 or more chronic illnesses?

(iii) Do you take 5 or more medications?

(iv) Have you been hospitalized in the past 3 months?

(2) Identify Cognitive and Functional Abilities. Use of cognitive, depression, and functional assessment tools (e.g., MiniCog Assessment, Geriatric Depression Scale, Activities of Daily Living, and Instrumental Activities of Daily Living assessments) may be particularly helpful with the patient's care assessment and plans for discharge [27-30]. Consider asking the following:

(i) Do you need help with bathing, dressing, shopping, and paying bills?

(ii) Do you feel sad?

(iii) Are you lonely?

(3) Obtain Detailed Social Support Information. It is important to call any possible contacts that may be beneficial in identifying care for elder orphans. This may include out-of-town family, friends, neighbors, physicians, and significant others. Furthermore, all resources and benefits available for the patient need to be identified. A social worker can assist with gathering some of the information. Consider asking the following:

(i) Who could help you in a crisis?

(ii) Do you have a long-term care policy?

(iii) Are you a veteran in the military?

(4) Create a Manageable and Realistic Treatment Plan. Individuals without support need to have treatment plans that can be achieved.

(5) Utilize Service Delivery to Home. For example, utilize home care, pharmacy, and food delivery services.

(6) Make Safety and Injury Prevention a Priority; Address Safety and Injury Issues. Consider asking the following:

(i) Have you fallen? 
TABLE 2: The table shows the prevalence of those at risk of becoming an elder orphan based on 2010 data. Prevalence was calculated by dividing the sum of the total individuals in the "unmarried, with children, but not in contact" tier and the "unmarried, without children" tier (the two biggest risk factors for becoming an elder orphan) by the total of respondents to the health and retirement study [4].

\begin{tabular}{|c|c|c|}
\hline Risk description & Number & $\begin{array}{c}\text { Percent (out of 22,034 } \\
\text { respondents) }\end{array}$ \\
\hline Unmarried, with children, but not in contact & 3,903 & $17.7 \%$ \\
\hline Unmarried, with children, but they are not in contact, and there are no siblings within 10 miles & 3,738 & $17.0 \%$ \\
\hline Unmarried, with children, but children live further than 10 miles away & 3,106 & $14.1 \%$ \\
\hline Unmarried, with children, but not within 10 miles, and there are no siblings within 10 miles & 48 & $0.2 \%$ \\
\hline Unmarried, without children & 1,071 & $4.9 \%$ \\
\hline Unmarried without children or siblings & 141 & $0.6 \%$ \\
\hline Totally unmarried, without children, or unmarried with children, not in contact & 4,974 & $22.6 \%$ \\
\hline
\end{tabular}

Total prevalence of at-risk individuals $=($ unmarried, with children, not in contact $)+($ unmarried without children $)=(3,903+1,071) / 22,034=22.6 \%$.

(ii) Do you have a gun in your home?

(iii) Are you driving? Did you experience any accidents? Do you wear your seatbelt regularly? Have you gotten lost while driving?

(7) Address Goals of Care and Advance Directives. By focusing on health-care proxy and living will, future resuscitation, mechanical ventilation, treatment, hospitalization, and even funeral and burial arrangement wishes may elicit support systems that exist. Consider asking the following:

(i) Do you have a health-care proxy or durable power of attorney for healthcare?

(ii) Do you have a living will?

(iii) Do you have a will for your belongings, property? Who has helped you with these?

(iv) Have you discussed future treatment, hospitalization, burial wishes, or arrangements with anyone or made future plans?

(8) Understand Privacy Issues (HIPAA). Health-care workers must be cognizant of privacy laws while understanding that the intent of reaching out to support systems is to assist in medical care and health advocacy. Health professionals must fully document that the purpose of outreach is for the safety and health of the individual, and, in so doing, privacy laws are respected but do not form a barrier to coordination of care.

(9) Assess Decision-Making Capacity and Involve the Individual as Much as Possible. Assess whether the person has the ability to make specific decisions, as capacity is valid solely on a case-by-case basis and based on a specific issue being decided on. Although a person may be failing in some cognitive abilities, it does not necessarily mean that they lack the ability to make certain health-care decisions [31].

(10) Determine If Guardianship Is Needed, and If So, Seek It. A guardianship is a legal relationship created when a person or institution is named in a will or assigned by the court to take care of incompetent adults. Consider contacting hospital legal or social work departments.
In Questions to Screen for Risk for Elder Orphan Status we outline key screening questions that can help healthcare providers identify individuals at risk of being elder orphans. These suggested questions can be self-administered or easily incorporated into other assessments completed by office assistants to help identify individuals at risk. Further studies on the effectiveness of these questions as a screening tool are needed. As shown in the Ten-Step Guide to Caring for an Elder Orphan, we have developed these 10-steps to assist providers in sorting through the complex physical and psychosocial issues that elder orphans face. We offer practical approaches to developing a multidisciplinary, holistic approach and care plan for these individuals to address a growing public health need.

Identifying these individuals prior to loss of function or admission into acute care facilities will help to expedite appropriate medical care, avert negative outcomes, and reduce the burden on the health system. Early identification of these at-risk individuals allows for care plans that can better meet the needs of the elder orphan.

We suggest the term elder orphan as a benevolent identifier for a group of individuals who find themselves in this difficult situation. We hope to incite awareness and action in the medical and social community to assist these older adults in society who are unable to complete instrumental activities of daily life and have no available caregivers, as well as those who are at risk of isolation and lacking support. Although other terms have been used to describe individuals who fall within the category of vulnerable older adults (e.g., the unbefriended elderly who are alone and lack decisionmaking ability), they have the potential to inadvertently stigmatize these individuals and often fall within legal realm. Thus, we resurrect the use of elder orphan as a benevolent and medical alternative to a more broad population of individuals who are alone and unsupported.

The purpose of the term, elder orphan, is for use in healthcare environment to highlight vulnerability and attract attention to the need for a care-giving and medical decisionmaking plan. What is not known is how use of this term might negatively impact the individual. It is the authors' hope that use of the term will lead to allocation of more resources for the individual. Further studies should investigate the impact 
of the use of the term on care on the individual and potential for unintended negative consequences such as stigmatization.

A limitation to our estimation of prevalence is that with the available data the physical and cognitive health of the subjects' relatives and friends is unknown, such as in Case 1, Ms. H. M. Future studies should analyze these variables in order to offer a more accurate prevalence. Moreover, as the data is based on marital status, it does not provide measurement of individuals with significant or domestic partners that are involved caregivers. More detailed analyses are needed to more accurately measure an at-risk elder orphan population.

The expected future increase in the number of individuals without support from children and/or spouses/partners, combined with a population that is living longer, poses an enormous challenge to both the health-care system and the community. Thus, further studies are needed to elucidate the exact prevalence of this population, the needs of this group, and the resources currently available to them. Moreover, a critical view of the risks of being an elder orphan must be delineated in order to more adequately prepare for and minimize them. In these future studies, care must be taken to examine the number, health status, and relationship of the subjects' existing family members, as well as their marital status and health-care advocates.

In addition, the services needed for this population should be scrutinized. This at-risk group requires access to a host of services in order to help them thrive independently in the community. Those identified as elder orphans should be educated about advanced directives and creating a plan of care far in advance of needing acute care. More importantly, they should receive assistance as needed and as available to implement and achieve a plan of care. A few simple measures can help stem catastrophe, and some possible resources needed for this population are the following:

(i) Community based aging resource centers and adult day care centers (community access to social services and senior organizations with a goal of preventing avoidable hospital admissions).

(ii) Community multidisciplinary teams to care for patients with medical, functional, social, and safety needs.

(iii) Public-private partnerships to help vulnerable populations, linking health-care teams with community and government agencies (e.g., social services, adult protective services, and senior agencies).

Based on our clinical experience and a literature review, we propose ten steps physicians and other providers should take into account to identify and help address the medical and psychosocial needs of elder orphans in the community, as outlined in Ten-Step Guide to Caring for an Elder Orphan.

\section{Conclusion}

The elder orphan population is an increasingly prevalent and at-risk demographic living precariously in the community. They often go unrecognized by health-care providers and the community alike, silently living in danger of medical crises.
The medical, public health, and general community need to become more aware of these individuals in order to protect and advocate for them. Our proposed screening questions in Questions to Screen for Risk for Elder Orphan Status and ten-step guide in Ten-Step Guide to Caring for an Elder Orphan can help when faced with caring for an older adult with no one. Further action is vital but steps, as outlined, could begin to address this growing population, identify the needs, raise awareness in order to mobilize public health and community resources, and prioritize development of caregiving and decision-making plans, so that these individuals are no longer hiding in plain sight.

\section{Disclosure}

A poster presentation of this paper was presented at the 2015 Annual Scientific Meeting of the American Geriatric Society.

\section{Competing Interests}

None of the authors listed have financial interests or conflict of interests to disclose relevant to the subject of this paper.

\section{References}

[1] N. R. Kleinfeld, The Lonely Death of George Bell, section A, The New York Times, 2015.

[2] P. Span, The Last 'Friend' You May Ever Need, section D, The New York Times, 2015.

[3] Tribune Media Wire Service, "Elderly man with cancer calls 911 because he has no food," May 2015, http://fox6now.com/ 2015/05/13/elderly-man-with-cancer-calls-911-because-he-hasno-food/.

[4] Health and Retirement Study, (Family Data) Public Use Dataset, Grant Number NIA U01AG009740, University of Michigan, Ann Arbor, Mich, USA; National Institute on Aging, 2010.

[5] J. Kunerth, Seniors Outliving Family, Becoming Elder Orphans, Chicago Tribune, 2003.

[6] R. Sherer, "Who will care for elder orphans," Geriatric Times, vol. 5, no. 1, 2004.

[7] J. Varner, “The elder orphans: who are they?" The Alabama Nurse, vol. 32, no. 3, pp. 19-21, 2005.

[8] D. G. Blazer, "Social support and mortality in an elderly community population," American Journal of Epidemiology, vol. 115, no. 5, pp. 684-694, 1982.

[9] T. E. Seeman, "Social ties and health: the benefits of social integration," Annals of Epidemiology, vol. 6, no. 5, pp. 442-451, 1996.

[10] R. Larson, "Being alone verus being with people: disengagement in the daily experience of older adults," Journals of Gerontology, vol. 40, no. 3, pp. 375-381, 1985.

[11] L. S. Dolen and D. J. Bearison, "Social interaction and social cognition in aging. A contextual analysis," Human Development, vol. 25, no. 6, pp. 430-442, 1982.

[12] B. B. Arnetz, T. Theorell, L. Levi, A. Kallner, and P. Eneroth, "An experimental study of social isolation of elderly people: psychoendocrine and metabolic effects," Psychosomatic Medicine, vol. 45, no. 5, pp. 395-406, 1983.

[13] C. M. Perissinotto, I. S. Cenzer, and K. E. Covinsky, "Loneliness in older persons: a predictor of functional decline and death," 
Archives of Internal Medicine, vol. 172, no. 14, pp. 1078-1083, 2012.

[14] D. Sorkin, K. S. Rook, and J. L. Lu, "Loneliness, lack of emotional support, lack of companionship, and the likelihood of having a heart condition in an elderly sample," Annals of Behavioral Medicine, vol. 24, no. 4, pp. 290-298, 2002.

[15] G. C. Wenger, R. Davies, S. Shahtahmasebi, and A. Scott, "Social isolation and loneliness in old age: review and model refinement," Ageing and Society, vol. 16, no. 3, pp. 333-358, 1996.

[16] M. G. Thompson and K. Heller, "Facets of support related to well-being: quantitative social isolation and perceived family support in a sample of elderly women," Psychology and aging, vol. 5, no. 4, pp. 535-544, 1990.

[17] J. A. Udell, P. G. Steg, B. M. Scirica et al., "Living alone and cardiovascular risk in outpatients at risk of or with atherothrombosis," Archives of Internal Medicine, vol. 172, no. 14, pp. 10861095, 2012.

[18] C. L. Johnson and D. J. Catalano, "Childless elderly and their family supports,” The Gerontologist, vol. 21, no. 6, pp. 610-618, 1981.

[19] L. J. Beckman and B. B. Houser, "The consequences of childlessness on the social-psychological well-being of older women," Journals of Gerontology, vol. 37, no. 2, pp. 243-250, 1982.

[20] N. G. Choi, "Patterns and determinants of social service utilization: comparison of the childless elderly and elderly parents living with or apart from their children," The Gerontologist, vol. 34, no. 3, pp. 353-362, 1994.

[21] Z. Zhang and M. D. Hayward, "Childlessness and the psychological well-being of older persons," Journals of Gerontology B: Psychological Sciences and Social Sciences, vol. 56, no. 5, pp. S311S320, 2001.

[22] N. Mahgoub and M. Lantz, "When older adults suffer the loss of a child," Psychiatric Annals, vol. 36, no. 12, pp. 877-880, 2006.

[23] J. M. Lee, S. Pilli, A. Gebremariam et al., "Getting heavier, younger: trajectories of obesity over the life course," International Journal of Obesity, vol. 34, no. 4, pp. 614-623, 2010.

[24] L. M. Monte and R. R. Ellis, "Fertility of Women in the United States: 2012," in Commerce USDo, U.S. Census Bureau, 2014.

[25] I.-F. Lin and S. L. Brown, "Unmarried boomers confront old age: a national portrait," Gerontologist, vol. 52, no. 2, pp. 153165, 2012.

[26] American Association of Retired P, United S. Aging/Parents \& Adult Children Together, A/PACT, Washington, D.C, USA, Federal Trade Commission, AARP, 1999.

[27] S. Borson, J. M. Scanlan, P. Chen, and M. Ganguli, “The MiniCog as a screen for dementia: validation in a population-based sample," Journal of the American Geriatrics Society, vol. 51, no. 10, pp. 1451-1454, 2003.

[28] J. I. Sheikh and J. A. Yesavage, "Geriatric Depression Scale (GDS): recent evidence and development of a shorter version," Clinical Gerontologist, vol. 5, no. 1-2, pp. 165-173, 1986.

[29] S. Katz, "Assessing self-maintenance: activities of daily living, mobility, and instrumental activities of daily living," Journal of the American Geriatrics Society, vol. 31, no. 12, pp. 721-727, 1983.

[30] M. P. Lawton and E. M. Brody, "Assessment of older people: self-maintaining and instrumental activities of daily living," The Gerontologist, vol. 9, no. 3, pp. 179-186, 1969.

[31] P. S. Appelbaum and T. Grisso, "Assessing patients' capacities to consent to treatment," The New England Journal of Medicine, vol. 319 , no. 25, pp. 1635-1638, 1988. 


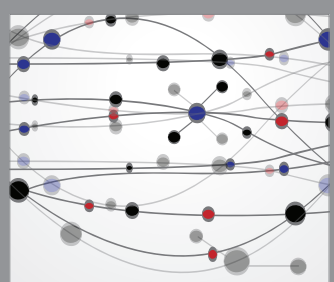

The Scientific World Journal
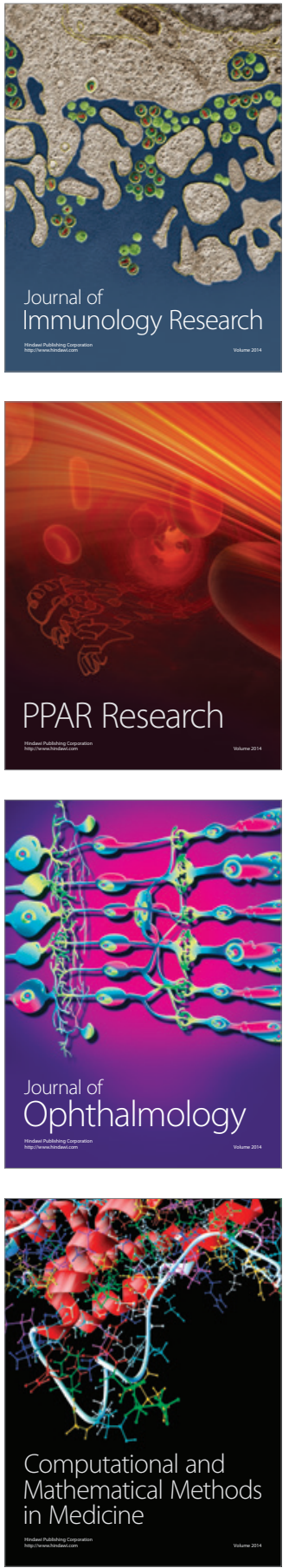

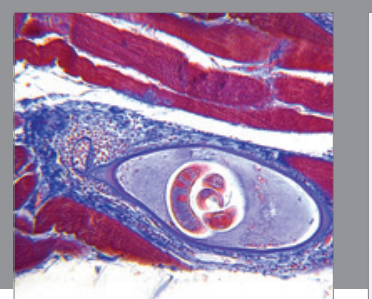

Gastroenterology Research and Practice

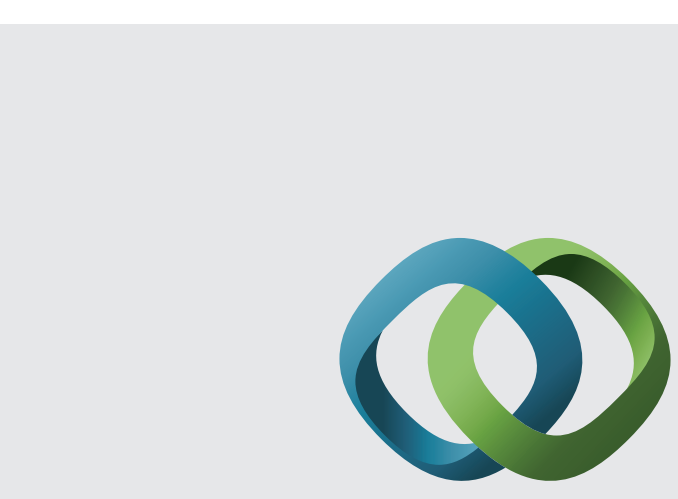

\section{Hindawi}

Submit your manuscripts at

http://www.hindawi.com
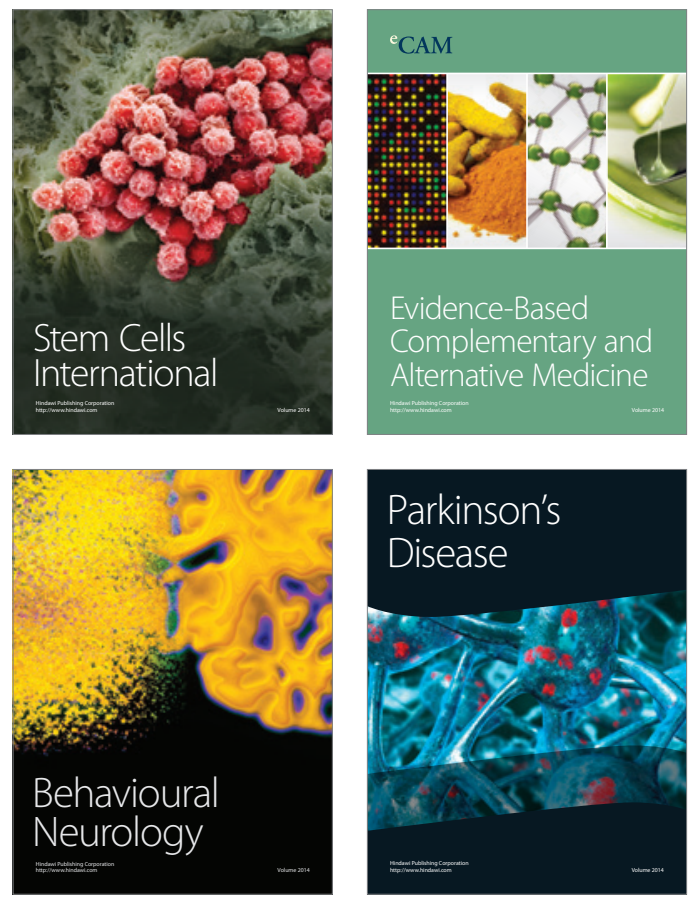
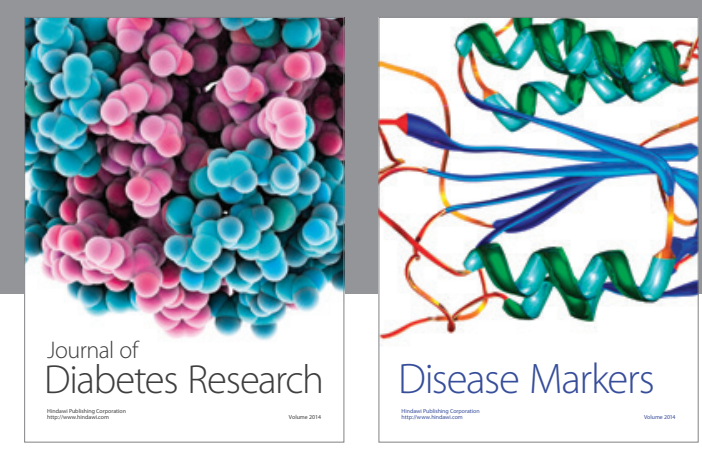

Disease Markers
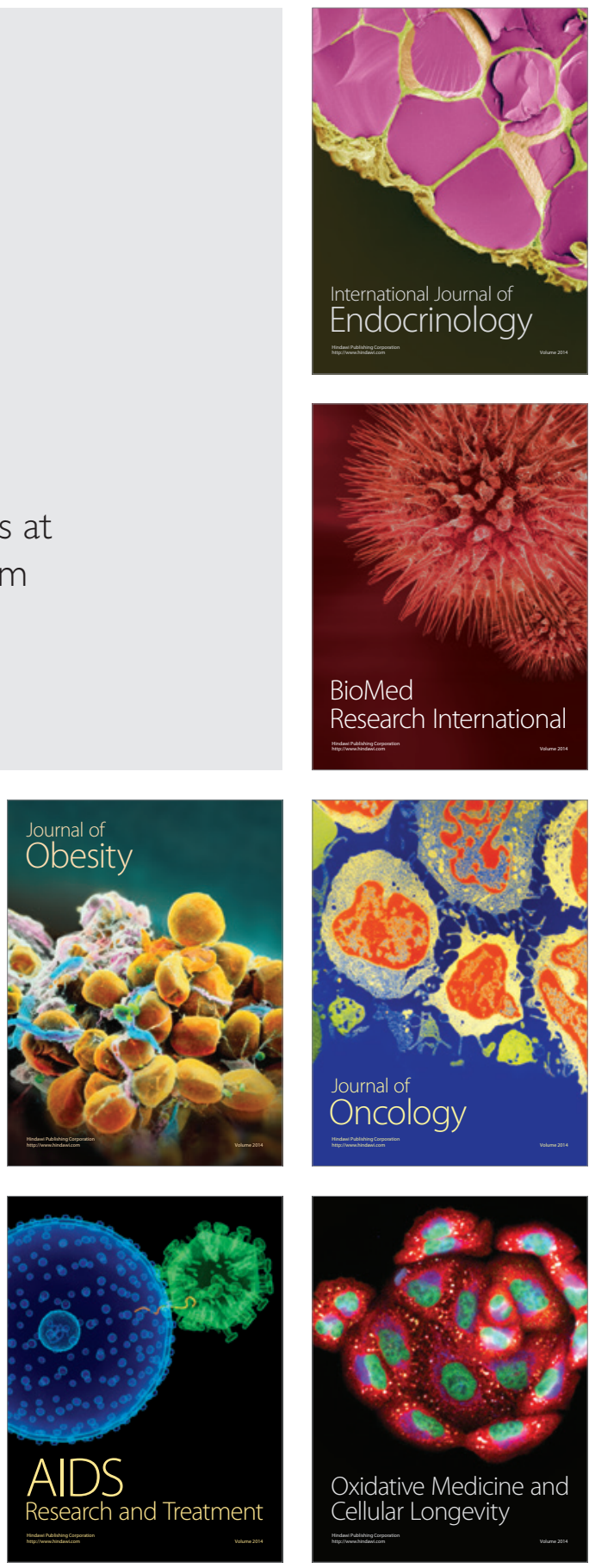